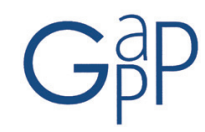

GESTIÓN Y ANÁLISIS DE POLÍTICAS PÚBLICAS, Nueva Época, nº 11 enero-junio 2014 ISSN: 1989-8991

DOI: http://dx.doi.org/10.24965/gapp.voi11.10175

\title{
El espacio catalán de relaciones laborales: breve reflexión sobre el modelo de participación institucional
}

\author{
Miguel Ángel Catalinas \\ Escuela de Administración Pública de Catalunya. Generalitat de Catalunya
}

miguel.catalinas@gencat.cat

Recibido: 10 enero 2014

Aceptado: 10 febrero 2014

\begin{abstract}
Resumen
La actual situación económica ha reabierto el debate sobre la reforma de la administración pública, concretamente desde la vertiente del gasto que realiza y el tipo de servicios que debe seguir prestando o no. Asimismo, se han promovido diversos estudios e informes que cuestionan la viabilidad de determinados organismos públicos, con el objetivo de buscar una mejor eficiencia y evitar duplicidades. En este sentido, se plantea la reflexión sobre tres organismos que forman parte del marco de relaciones laborales de Catalunya (EI Consejo de Relaciones Laborales, El Tribunal Laboral de Catalunya y el Consejo de Trabajo, Económico y Social de Catalunya), con el fin de explorar posibles modelos institucionales alternativos, que se construiran aplicando la metodología de los MOE's (modelos de orientación estratégica), que se ajusten mejor a la realidad económica y laboral y que, en todo caso, den respuesta a la necesidad de establecer estructuras organizativas más eficientes.
\end{abstract}

Palabras clave

Concertación social, resolución extrajudicial de conflictos laborales, Consejo de Relaciones Laborales, Tribunal Laboral de Catalunya, Consejo de Trabajo, Económico y Social de Catalunya

\section{Catalan labour relations framework: brief reflection on institutional frame}

\section{Abstract}

The current economic situation has reopened the debate on the reform of public administration, particularly from the perspective of the expenditure carried out and the type of services that must continue being offered or not. Several studies and reports have been also tackling the discussion on the viability of certain public bodies in order to achieve better efficiency and avoid duplications. We focus our study on three institutions that comprise the institutional frame of labour relations in Catalonia (Labour Relations Board, the Labour Court of Catalonia, Economic and Social Council of Catalonia), models will be designed using "MOE"'s methodology (Strategic Orientation Models) in order to explore alternative institutional frames that may fit properly in economic and labor current context and also respond to requirements for more efficient organizational structures.

\section{Keywords}

Social partnership, extrajudicial resolution of labor disputes, Labour Relations Board, Labour Court of Catalonia, Labor Council, Economic and Social de Catalonia.

\section{INTRODUCCIÓN}


La actual crisis económica ha obligado a reflexionar sobre diversos aspectos de nuestra vida cotidiana y cuestionar conductas que posiblemente nunca habríamos cuestionado. En el ámbito del sector público, la deuda acumulada y, sobretodo, el encarecimiento de su financiación, debido a las incerteza sobre el crecimiento y fortaleza económica del país, unido a una notable caída de los ingresos de las arcas públicas, ha provocado reflexionar sobre la viabilidad de diversos servicios y políticas públicas, analizar los gastos que ocasionaban y valorar si eran necesarios. En definitiva, ha generado una revisión de todo lo que se hacía hasta el momento, buscando aquello que fuera accesorio y que, por tanto, supusiera un ahorro para el erario público.

Este diagnóstico, en buena medida, queda recogido en el documento de la Comisión para la Reforma de las Administraciones Pública (CORA)', creada por el Gobierno con el fin de detectar posibles ineficiencias y duplicidades en el conjunto de las administraciones públicas del Estado. De hecho, se establece la Subcomisión de Administración Institucional para "analizar la distinta tipología de entes que la componen, revisar el marco normativo y los modelos que en él se identifican como óptimos y plantear las modificaciones que convengan en la lista de entitades existentes" ${ }^{2}$. En este sentido y aunque el ámbito de la Subcomisión se centra en el Sector Público Estatal, podemos trasladar este cometido al ámbito de la Generalitat de Catalunya y plantear un caso concreto.

De acuerdo con el Estatuto de Autonomía de Catalunya (art. 45)³, se establece que,

"la Generalitat tiene que promover la creación de un espacio catalán de relaciones laborales establecido en función de la realidad productiva y empresarial específica de Catalunya y de sus agentes sociales, en el cual tienen que estar representadas las organizaciones sindicales y empresariales y la Administración de la Generalitat. En este marco, los poderes públicos tienen que fomentar una práctica propia de diálogo social, de concertación, de negociación colectiva, de resolución extrajudicial de conflictos laborales y de participación en el desarrollo y la mejora del entramado productivo".

Si analizamos esta referencia, podemos detectar diferentes elementos característicos de este espacio. Por un lado y como elemento subjetivo, identifica la necesidad de que estén representadas las organizaciones sindicales y empresariales ${ }^{4}$ y la Administración de la Generalitat. Por otra parte y como elementos objetivos, establece que:

- Tiene que posibilitar una práctica propia de diálogo social, de concertación y de negociación colectiva.

- Tiene que fomentar la resolución extrajudicial de conflictos laborales.

- Tiene que permitir la participación en el desarrollo y la mejora del entramado productivo.

Llegados a este punto, podríamos plantearnos si, efectivamente, se ha construido este espacio propio. La respuesta, desde un punto de vista institucional, sería afirmativa. Así, si observamos el conjunto de instituciones existentes en Catalunya, podríamos identificar los tres elementos antes mencionados con los siguientes organismos:

- El Tribunal Laboral de Catalunya (TLC), con respecto a la resolución extrajudicial de conflictos laborales.

- El Consejo de Trabajo, Económico y Social de Catalunya (CTESC), en relación a la participación en el desarrollo y la mejora del entramado productivo.

- El Consejo de Relaciones Laborales (CRL), con respecto al diálogo social, la concertación y la negociación colectiva.

En los tres casos encontramos representación de las organizaciones empresariales y sindicales más representativas de Catalunya pero, lógicamente, mantienen diferencias relevantes respecto de su naturaleza, organización $y$, por supuesto, funciones.

Así, la reflexión que se llevará a cabo es presentar y comentar brevemente estos aspectos (estructura organizativa, funciones, recursos) y proponer, si procede, un nuevo modelo institucional con el objetivo de:

1. Racionalizar su estructura.

2. Convertirse en un modelo que de respuesta de forma eficiente a las necesidades de la sociedad y economía catalana.

3. Potenciar su imagen y visualización dentro del espacio catalán de relaciones laborales.

$1 \quad$ Apartado 1 , página 12

2 Apartado VII, página 209

3 Ley Orgánica 6/2006, de 19 de julio, de reforma del Estatuto de Autonomía de Cataluña. Cabe añadir que en el anterior Estatuto de Autonomía de 1979, no se recoge la promoción de tal espacio.

4 Hay que entender organizaciones sindicales y empresariales más representativas de Catalunya. GESTIÓN Y ANÁLISIS DE POLÍTICAS PÚBLICAS, Nueva Época, nº 11 enero-junio 2014 ISSN: 1989-8991 - DOI: 10.24965/gapp.v0i11.10175 
Para llevar a cabo este objetivo, se utilizará la metodología de los MOEs (Modelos de Orientación Estratégica)' que posibilita el rediseño organizativo de una organización, partiendo de un diagnóstico y, con la interacción de dos ejes dicotómicos previamente establecidos, la construcción de cuatro modelos organizativos y una propuesta final sobre uno de ellos. No obstante y dado que el caso que trataremos reflexiona sobre un modelo institucional con diferentes organizaciones y no sobre una organización en concreto, se adaptará su aplicación. Así pues, se requiere identificar dos variables clave para establecer los ejes que habilitarán la construcción de los diferentes modelos. En este sentido en base a los objetivos antes enumerados (sobretodo el 2 y 3 ) y atendiendo a que dichas variables deben ofrecer un criterio orientador tanto para la estructura como para las dinámicas de funcionamiento que puede tomar la organización para realizar con éxito la misión y los objetivos que tiene predefinidos ${ }^{6}$, se proponen las siguientes:

- Grado de presencia de las organizaciones sindicales y empresariales en la composición de los órganos de los organismos.

- Grado de estructuración del modelo, con una mayor o menor integración/concentración de las estructuras existentes.

Por último, se presentará un apartado donde se apuntaran las actuaciones más relevantes que se deberían llevar a cabo con el fin de aplicar el modelo propuesto.

En este sentido, cabe advertir que el objetivo fundalmental del presente documento es realizar una reflexión y promover un debate sobre si la actual estructura institucional del Espacio Catalán de Relaciones Laborales se pueden reformular para convertirlo en un estructura más eficiente, a partir de una breve presentación de los organismes actuales y utilizando una metodología concreta. Por tanto, no pretende revisar fundamentos teóricos ni establer un estudio comparado de modelos de participación institucional en otras Comunidades Autónomas, que probablemente sería materia suficiente para realizar otro artículo.

\section{LAS INSTITUCIONES}

\subsection{El Tribunal Laboral de Catalunya}

Con la firma del Acuerdo Interprofesional de Catalunya (AIC) entre la patronal Fomento del Trabajo Nacional (Fomento) y los sindicatos UGT y CCOO, se crea en 1990 el tribunal laboral de conciliación, mediación y arbitraje, con el objeto de establecer un sistema extrajudicial y extraadministrativo de solución de la conflictividad laboral. En 1992 y con el fin de adquirir la capacidad jurídica necesaria para el desarrollo de las actividades del tribunal, las partes firmantes del Acuerdo constituyen la fundación privada Tribunal Laboral de Catalunya.

En el patronato, como órgano de gobierno y representación de la Fundación se encuentran representadas las tres organizaciones antes mencionadas (dos miembros de Fomento, uno de UGT y uno de CCOO) y la Generalitat de Catalunya, mediante el titular de la Dirección General de Relaciones Laborales y Calidad en el Trabajo del Departamento de Empresa y Empleo, que asume la Presidencia de lo mismo. En este sentido, el Departamento subvenciona la financiación de los gastos de funcionamiento de la Fundación?

De acuerdo con su reglamento ${ }^{8}$, la Fundación, además de otras funciones, como las de intervención en los conflictos de intereses que le sean propios, actúa como órgano de conciliación y mediación a los efectos de intentar, de forma previa a la demanda judicial, la conciliación individual, plural o colectiva establecida, respectivamente, en los artículos 63 y 156 de la Ley 36/2011, de 10 de octubre, reguladora de la jurisdicción social.

Con independencia del tipo de conflicto a gestionar (jurídicos o de intereses), su actividad se canaliza principalmente en tres tipos de procedimientos: conciliaciones, mediaciones y arbitrajes, siendo en todos los casos, requerida la sumisión de las partes:

- Conciliación: conjunto de acciones que llevan a cabo los miembros de la Delegación del Tribunal con el fin de obtener un acta de conciliación con avenencia de las partes.

- Mediación: consiste, una vez no ha habido acuerdo en la conciliación, a someter el conflicto a la Comisión de Mediación del Tribunal, a fin de que ésta formule una propuesta mediadora que posibilite la avenencia de las partes.

5 Ramió, C.; Salvador, M. “Los modelos de orientación estratégica (MOEs): una adaptación del enfoque estratégico para el diseño organizativo en las Administraciones públicas". 1999

6 Ramió, C.; Salvador, M., 1999: página 92.

7 Apartado 3 del "manifiestan" del convenio de colaboración entre el Departamento de Empresa y Empleo y Fundación Privada Tribunal Laboral de Catalunya (2012)

8 http://www.tribulab.cat/reglament/ GESTIÓN Y ANÁLISIS DE POLÍTICAS PÚBLICAS, Nueva Época, n 11 enero-junio 2014 ISSN: 1989-8991 - DOI: 10.24965/gapp.v0i11.10175 
- Arbitraje: consiste en la sumisión expresa de las partes a la decisión (laudo) de uno o diversos árbitros o del propio Tribunal sobre el conflicto presentado. En todos los casos, el laudo tendrá carácter vinculante para las partes.

La Fundación estructura sus actuaciones mediante las delegaciones, las comisiones de mediacionesª las comisiones técnicas y el cuerpo de árbitros. Asimismo, cuenta con un Patronato con un presidente y un gerente.

- Presidente: Recae en la persona titular de la Dirección General de Relaciones Laborales y Calidad en el Trabajo del Departamento de Empresa y Empleo y sus funciones son, básicamente, de representación legal de la Fundación y de convocatoria y presidencia de las reuniones del Patronato.

- Patronato: es el órgano de gobierno y de administración de la Fundación, la representa y gestiona, y asume todas las facultades y funciones necesarias para la consecución de los fines fundacionales. Está constituido por cinco miembros: dos miembros de Fomento, uno de UGT, uno de CCOO y uno de la Generalitat de Catalunya.

- Gerente: ejerce la función de dirección de la gestión ordinaria, tanto económica como administrativa, de la Fundación y de la ejecución de los acuerdos del Patronato.

- Delegaciones: es órgano que se encarga del conocimiento de los conflictos que se canalizan mediante el procedimiento de conciliación. Están compuestas por cuatro miembros, en el caso de los conflictos colectivos o de dos miembros, en los procedimientos de carácter individual y conflictos colectivos o plurales que afecten en una empresa con menos de 30 trabajadores. En todos los casos, los miembros tienen que formar parte del cuerpo de conciliadores del Tribunal.

- Comisiones de mediaciones: se encargan del conocimiento de los conflictos que se canalizan mediante el procedimiento de mediación. Están compuestas por cuatro miembros, en el caso de los conflictos colectivos o de dos miembros, en los procedimientos de carácter individual y conflictos colectivos o plurales que afecten en una empresa con menos de 30 trabajadores. En todos los casos, los miembros tienen que formar parte de la Comisión de Mediación del Tribunal, integrada de forma permanente por veintiséis mediadores designados por las organizaciones.

- Comisiones técnicas: es el órgano de peritaje, dictamen o informe de las delegaciones, de la Comisión de Mediación o del cuerpo de árbitros, constituidas en base a algún aspecto que se crea indispensable para determinar, en función del objeto del conflicto, los aspectos técnicos imprescindibles en materia de organización del trabajo, situación económica-financiera de empresas, prevención de riesgos laborales, igualdad de oportunidades, no discriminación en razón de género o cualquier otra materia que requiera la intervención y dictamen de expertos técnicos. Actualmente existen tres comisiones técnicas: la de Organización del Trabajo, la de Economía y Previsión Social Complementaria y la de Seguridad y Salud Laboral.

- Cuerpo de árbitros: está constituido por los árbitros designados por las diferentes organizaciones, manteniendo siempre la paridad representativa entre organización empresarial y sindicato.

\section{Esquema organizativo}

- Presidencia

- Patronato

- Gerente

- Delegaciones

- Comisión de mediación - cuerpo de conciliadores

- Comisiones técnicas

- Organización del Trabajo

- Economía y Previsión Social Complementaria.

- Seguridad y Salud Laboral.

9 La composición de estos dos órganos es paritaria entre las organizaciones empresariales y sindicales (http://www.tribulab.cat/reglament/ capitol-2-el-tribunal-laboral-estructura-i-funcionament). Si bien inicialmente, estas serían UGT, CCOO y Fomento, tras las adhesiones al Acuerdo Interprofesional de Catalunya (art. 3) de las organizaciones Unión Sindical Obrera de Catalunya (USOC), Pequeña y la Mediana Empresa de Catalunya (PIMEC) y Federación de Empresarios de la Pequeña y la Mediana Empresa de Catalunya (FEPIME), estas también podrían formar parte de estos órganos (página 3 y 12, Guía Rápida, http://www.tribulab.cat/wp-content/uploads/2014/02/TLC2013CATALAx.pdf) 


\section{- Cuerpo de árbitros}

\subsection{El Consejo de Trabajo, Económico y Social de Catalunya}

EI CTESC, si bien sus antecedentes se remontan a los inicios del siglo XX, se crea como tal con la aprobación de la Ley 3/1997, de 16 de mayo, lo que supone la agrupación en un solo organismo del Consejo de Trabajo, el Consejo de Seguridad y Salud Laboral y el Consejo Catalán de Formación Ocupacional y Colocación. Así, el nuevo Consejo asume las funciones consultivas y de asesoramiento en el Gobierno en materias sociolaborales, ocupacionales y socioeconómicas, y se convierte en el órgano de participación institucional. No obstante, mediante la Ley 7/2005, de 8 de junio, desaparece esta última función de participación institucional (que recogerá en el 2007 el Consejo de Relaciones Laborales, como se verá posteriormente) ${ }^{10}$.

El Consejo es un ente de derecho público, con personalidad jurídica propia, plena capacidad y autonomía orgánica y funcional para el cumplimiento de sus finalidades, y se encuentra adscrito al departamento competente en materia de trabajo (actualmente, el Departamento de Empresa y Empleo) ${ }^{11}$. En este sentido, el Consejo formula anualmente una propuesta del presupuesto con el fin de ser incluida en el anteproyecto de presupuesto de la Generalitat de Catalunya ${ }^{12}$.

El Consejo está compuesto por miembros de tres Grupos diferentes: Grupo Primero, representantes de organizaciones sindicales (UGT y CCCO); Grupo Segundo, representantes de organizaciones empresariales (Fomento y PIMEC) y Grupo Tercero, representantes del sector agrario (Unión de Payeses), marítimo pesquero (Federación Nacional Catalana de Cofradías de Pescadores), economía social (Confederación de Cooperativas de Catalunya y Federación de Sociedades Laborales de Catalunya) y expertos en materia de las competencias del Consejo.

Por último, hay que señalar que en el año 2006 el Consejo se convierte en órgano estatutario al quedar recogido en el Estatuto de Autonomía de Catalunya (art. 72.2).

Como se ha indicado anteriormente, el Consejo es un órgano consultivo y de asesoramiento del Gobierno de la Generalitat. En este sentido, entre sus principales funciones encontramos:

a) Emitir dictamen con carácter preceptivo, no vinculante y previo a la tramitación correspondiente, sobre los anteproyectos de ley (excepto el de presupuestos), los proyectos de decreto legislativos y los proyectos de decreto (si así se determina) que regulan materias socioeconómicas, laborales y de empleo, competencia de la Generalitat.

b) Emitir dictamen sobre los planes y los programas que el Gobierno puede considerar que tienen una trascendencia especial en la regulación de las materias propias del Consejo, y también sobre los asuntos que, facultativamente, le sometan a consulta el Gobierno o los consejeros o consejeras o bien las organizaciones que integran el Consejo.

c) Elaborar propuestas, informes o estudios a solicitud del Gobierno o los consejeros o consejeras o por iniciativa propia sobre materias del ámbito de sus competencias.

d) Elaborar y enviar al Gobierno una memoria en que se reflejen sus consideraciones con respecto a la situación socioeconómica y laboral de Catalunya, y una reflexión sobre la posible evolución de esta situación.

e) Elaborar y enviar al Gobierno un informe anual sobre la situación de los trabajadores autónomos en Catalunya.

Con respecto a la estructura organizativa del Consejo, éste se compone de dos órganos unipersonales: uno representativo-institucional (Presidencia) y uno ejecutivo (Secretaría Ejecutiva), así como un órgano de representación plural y paritario entre los diferentes Grupos de representación (Pleno). Asimismo, también existe la Comisión Ejecutiva, como órgano ejecutivo colegiado, además de las comisiones de trabajo.

- Presidencia: su titular es nombrado y separado por el Gobierno, a propuesta conjunta del consejero del Departamento de Empresa y Empleo y de Economía y Conocimiento, con consulta previa de los grupos de representación del Consejo y con el apoyo de dos tercios de los miembros de lo mismo. Dentro de sus funciones, destaca la de representación legal del Consejo, dirigir la actividad o convocar y presidir las reuniones de los diferentes órganos con representación de los grupos.

- Vicepresidencias: las dos Vicepresidencias son ocupadas por sendos miembros de los Grupos Primero y Se-

$10 \mathrm{http}: / /$ www.ctescat.cat/el consell/qui som/historia/index.html

11 Ver Ley 7/2005, de 8 de junio, del Consejo de Trabajo, Económico y Social de Catalunya.

12 Desde los presupuestos de la Generalitat de Catalunya para el año 2006, cuenta con presupuesto propio en el subsector "Generalitat de Catalunya".

GESTIÓN Y ANÁLISIS DE POLÍTICAS PÚBLICAS, Nueva Época, n 11 enero-junio 2014 ISSN: 1989-8991 - DOI: 10.24965/gapp.v0i11.10175 
gundo y sus funciones son de sustitución del presidente o de delegación de funciones de lo mismo.

- Secretaría Ejecutiva: es el órgano que gestiona y garantiza el funcionamiento ordinario del Consejo. Se nombra y separa por el Gobierno, a propuesta conjunta del consejero del Departamento de Empresa y Empleo y de Economía y Conocimiento, con consulta previa de los grupos de representación del Consejo y con el apoyo de dos tercios de los miembros de lo mismo.

- Comisión Ejecutiva: Bajo la dirección del presidente y la asistencia de la secretaria ejecutiva, está formada por dos miembros de cada Grupo y entre sus funciones destaca la elaboración y aprobación del anteproyecto de presupuesto, la elaboración del proyecto de memoria anual o la elaboración del plan de trabajo anual.

- Pleno: Bajo la dirección del presidente, integra los treinta y seis miembros del Consejo, según los diferentes grupos: Grupo Primero, doce miembros en representación de las organizaciones sindicales; Grupo Segundo, doce miembros en representación de las organizaciones empresariales y Grupo Tercero, seis miembros en representación del sector agrario, pesquero marítimo y de la economía social y seis miembros en calidad de expertos en materias competencia del Consejo. Entre sus funciones, destaca la emisión de los dictámenes, la aprobación de los informes, propuestas y estudios o aprobar el presupuesto anual.

- Comisiones de trabajo: son los órganos encargados de la elaboración de las propuestas de dictámenes, estudios e informes. Se encuentran compuestas por doce miembros, de forma proporcional a los diferentes Grupos y están dirigidas por el presidente del Consejo y asistidas por la secretaria ejecutiva. Actualmente existen cuatro comisiones de trabajo: Economía y Fiscalidad y Unión Europea, Mercado de Trabajo y Políticas Sociales, Políticas Sectoriales y Desarrollo Territorial y Medio Ambiente.

Esquema organizativo

- Presidencia

- Vicepresidencias

- Secretaría Ejecutiva

- Pleno

- Doce miembros del Grupo Primero

- Doce miembros del Grupo Segundo

- Doce miembros del Grupo Tercero

- Comisión Ejecutiva

- Comisiones de trabajo

- Economía y Fiscalidad y Unión Europea

- Mercado de Trabajo y Políticas Sociales

- Políticas Sectoriales

- Desarrollo Territorial y Medio Ambiente

\subsection{EL CONSEJO DE RELACIONES LABORALES}

El CRL se creó mediante la Ley $1 / 2007$, del 5 de junio, dando respuesta, tal y como recoge el preámbulo de la misma, a la "doble necesidad de facilitar y racionalizar el diálogo social, por un lado, y de dar cobertura con carácter estable a la participación institucional de los agentes sociales", habida cuenta que con la reforma del CTESC, se elimina el espacio de concertación y participación social que hasta entonces existía con la presencia de representantes de la Generalitat'1'. Así pues, se convierte en un órgano de participación institucional, de diálogo y de concertación social en materia de relaciones laborales entre las organizaciones sindicales y empresariales más representativas de Catalunya y la Administración de la Generalitat. En este sentido, se encuentran representados los sindicatos UGT y CCOO y las patronales Fomento y Pequeña y Mediana Empresa de Catalunya (PIMEC) ${ }^{14}$.

13 En el preámbulo de la Ley $7 / 2005$, de 8 de junio, del Consejo de Trabajo, Económico y Social de Cataluña, se destaca la voluntad de reformar el organismo con dos finalidades: reforzar y profundizar la función consultiva y de asesoramiento al Gobierno propia de este organismo (eliminando el Grupo Cuarto, de representantes de la Generalitat) y remitir la función de participación institucional que hasta hoy ha ejercido a las instancias correspondientes, de acuerdo con la regulación que se apruebe.

14 Ver “El Consejo: conózcanos”, http://crl.gencat.cat

GESTIÓN Y ANÁLISIS DE POLÍTICAS PÚBLICAS, Nueva Época, nº 11 enero-junio 2014 ISSN: 1989-8991 - DOI: 10.24965/gapp.v0i11.10175 
Así, actúa con total autonomía e independencia en el ejercicio de sus funciones, y se encuentra adscrito al departamento competente en materia laboral de la Administración de la Generalitat que, actualmente, sería el Departamento de Empresa y Empleo. No obstante y en relación con su naturaleza jurídica, hay que indicar que es un órgano administrativo colegiado y, por lo tanto, no dispone de personalidad jurídica propia. En este sentido, el Consejo formula anualmente una propuesta del presupuesto con el fin de ser incluida en el anteproyecto de presupuesto del Departamento de Empresa y Empleo's.

Con respecto a las funciones, de acuerdo con el artículo 3 de la Ley 1/2007, serían:

a) Impulsar el diálogo entre las organizaciones sindicales y empresariales y entre éstas y la Administración de la Generalitat en materia de relaciones laborales, especialmente con respecto a la mejora y la racionalización de la negociación colectiva, la contratación laboral, la contratación y la subcontratación de obras y servicios, la igualdad y la no discriminación, la seguridad y la salud laboral, y la Inspección de Trabajo y Seguridad Social, por medio de la racionalización de las instancias de diálogo social existentes, para hacer efectivo el espacio catalán de relaciones laborales.

b) Garantizar y hacer efectiva la participación institucional en el control y el seguimiento de las materias de relaciones laborales.

c) Conocer las líneas estratégicas de actuación en materia de relaciones laborales presentadas por el departamento competente en esta materia de forma anual, y formular propuestas de actuación en materia de relaciones laborales para promover acuerdos entre las partes.

d) Ser consultado sobre los programas de actuación inspectora en Catalunya y recibir información sobre el seguimiento de dichos programas.

e) Fomentar y mejorar los diversos elementos que intervienen en la negociación colectiva en Catalunya, con el objetivo de impulsarla, orientarla y atender situaciones y circunstancias generales o particulares que puedan requerir atención y solución. Esta función incluye diversas actuaciones que tienen que ser aglutinadas y llevadas a cabo por la Comisión de Convenios Colectivos, como órgano especializado del Consejo.

La estructura organizativa del Consejo está basada en una relación de órganos unipersonales de tipo representativo institucional (Presidencia), directivo (Vicepresidencia) y ejecutivo (Secretaría General), así como un órgano de representación y, en este caso, paritario (Pleno). Asimismo, también existe la mesa de coordinación, como órgano ejecutivo colegiado, además de las comisiones y grupos de trabajo con el fin de facilitar y agilizar la tarea del Consejo.

- Presidencia: Recae en la persona titular del departamento competente en materia laboral de la Generalitat que, actualmente, sería el consejero del Departamento de Empresa y Empleo. En este sentido, la normativa sólo le atribuye exclusivamente "la presidencia de las sesiones a las que asiste", lo que supone que es un órgano eminentemente de representación institucional.

- Vicepresidencia: su nombramiento lo acuerda el Gobierno a propuesta del presidente del Consejo. Sus funciones son ejecutivas y de representación legal del Consejo.

- Secretaría General: es el órgano que gestiona y garantiza el funcionamiento ordinario del Consejo. Su puesto de trabajo es funcionarial, con rango asimilado a subdirector/a general y, como tal, se provee por el sistema de libre designación, siendo nombrado y separado por el consejero de Empresa y Empleo.

- Mesa de Coordinación: este órgano creado por el Pleno, está compuesto por un representante de la Generalitat y un representante de cada una de las organizaciones sindicales y empresariales presentes en el Consejo. Sus funciones son de tipo organizativo y ejecutivo, como la concreción de los puntos del orden del día de las reuniones del Pleno.

- Pleno: es el órgano que integra todos los miembros del Consejo y donde se visualiza la representación de las organizaciones sindicales y empresariales más representativas. En este sentido, está compuesto por el presidente o presidenta, el secretario o secretaria general y veinticuatro miembros, ocho en representación de la Administración de la Generalitat (incluye el titular de la Vicepresidencia), seis por parte de Fomento, cuatro por parte de UGT, cuatro parte de CCOO y dos por parte de PIMEC.

$15 \quad$ Art. 13.2 de la Ley $1 / 2007$. 
Entre sus funciones, destaca la aprobación del plan anual de trabajo del Consejo, de las normas de funcionamiento, del anteproyecto de presupuesto, así como conocer y formular las líneas estratégicas de actuación del Departamento de Empresa y Empleo en materia de relaciones laborales.

- Comisiones y grupos de trabajo: son los órganos de diálogo y participación institucional más operativos y de trabajo. Actualmente, existen ocho comisiones y tres grupos de trabajo, de diferentes ámbitos temáticos. Su composición respeta la representatividad y proporción de los grupos del Pleno.

\section{Esquema organizativo}

- Presidencia

- Vicepresidencia

- Secretaría General

- Mesa de coordinación

- Pleno

- Ocho miembros en representación de la Administración de la Generalitat

- Ocho miembros en representación de las organizaciones sindicales más representativas.

- Ocho miembros en representación de las organizaciones empresariales más representativas

- Comisiones

- Convenios Colectivos

- Comisión Técnica de Negociación Colectiva

- Seguimiento de la Contratación Laboral

- Seguridad y Salud Laboral

- Igualdad y del Tiempo de Trabajo

- Responsabilidad Social

- Inspección y Seguridad Social

- Observatorio del Trabajo

- Grupos de Trabajo

- Estrategia Catalana de Seguridad y Salud Laboral

- Acuerdo de Medidas de Empleo Juvenil

- Responsabilidad Social

\section{ANÁLISIS COMPARATIVO}

Una vez presentadas las tres instituciones y de forma previa a abordar la formulación de los diferentes modelos, realizaremos una breve reflexión comparativa sobre los aspectos destacados anteriormente de cada organización (naturaleza, funciones y estructura y organización), que a buen seguro nos será de gran utilidad para la comprensión del punto siguiente.

Uno de los elementos más diferenciadores de las tres instituciones es su naturaleza jurídica. El CRL, a pesar de estar regulado por ley, es un órgano administrativo sin personalidad jurídica propia. Este aspecto llama la atención, pues dicha regulación (por ley) posibilitaría que se hubiera configurado con dicha personalidad. En todo caso, dado que una unidad administrativa tiene menor autonomía, hace suponer que la intención del legislador era la de crear un órgano relativamente subordinado a la Administración. Prueba de ello es que, tal y como se ha visto, sus máximos órganos recaen el miembros de la Generalitat y no cuenta con un presupuesto propio e independiente al del Departamento de Empresa y Empleo.

Por otra parte, el CTESC sí que dispone de personalidad jurídica propia, lo que supone una situación bien diferente a la del CRL y que se visualiza, por ejemplo, en la disposición de un presupuesto propio. Asimismo, hay 
que indicar que tienen representación otras organizaciones (las del Grupo Tercero), además de las empresariales y sindicales más representativas. Ello se debe de la voluntad de reforzar, por un lado, la participación de los agentes económicos y sociales ${ }^{16}$, a efectos de poder conseguir, con una mayor enriquecimiento, la función del Consejo de asesorar al Gobierno en materia socioeconómica.

Por último, el TLC, como fundación privada, tiene plena autonomía de gestión y de actuación, presupuesto propio y, lógicamente personalidad jurídica propia. No obstante y atendiendo a la composición, encontramos que el Tribunal es la institución con menor implicación de la Generalitat, dado que no participó en la creación de la Fundación (se constituyó por Fomento, CCOO y UGT, de ahí su naturaleza privada) y su representación es minoritaria en el patronato de la misma (un único representante versus los dos de Fomento, uno de CCOO y uno de UGT). Probablemente, esta asimetría podría ser la composición más equilibrada en una situación un tanto insólita: una fundación privada, (que no requeriria, por tanto, tener representación de una administración pública), pero que tiene financiación pública, tal y como se ha indicado en el punto 2.1. También hay que destacar que, a diferencia de las otras dos instituciones, PIMEC no forma parte del Patronato de la Fundación, debido a que en el momento de su constitución (1992), esta organización empresarial no existía ${ }^{17}$.

Por contra en los dos Consejos, la representatividad de la Generalitat se visualiza en la competencia para nombrar a ambos presidentes, los cargos de responsabilidad ejecutiva y operativa, así como, en el caso del CRL, una representación paritaria ente los diferentes órganos. Todo hace que la construcción de los diferentes modelos esté condicionada a esta composición y, sobre todo, si pensamos en escenarios donde haya una mayor unificación estructural, donde requerirá una mayor negociación con las otras organizaciones representadas.

En relación con las funciones, podríamos hablar de una clara diferenciación entre las tres instituciones. El TLC, por ejemplo, se configura claramente como una instancia de mediación y resolución de conflictos laborales ${ }^{18}$. Por otra parte, el CRL se convierte en el órgano de participación institucional y concertación social, funciones que, hasta el año 2005, residían en el CTESC. Esta modificación normativa también comportó la desaparición del grupo de representados del Gobierno, lo que ha dado una mayor independencia en el ejercicio de sus funciones de asesoramiento y de emisión de informes, estudios y dictámenes.

\section{POSIBLES MODELOS INSTITUCIONALES}

Con el fin de formular los modelos, estableceremos las dos variables con cuya combinación podremos definir a los cuatro modelos institucionales. Así y de acuerdo con las características de las diferentes organizaciones comentadas, proponemos las siguientes variables o ejes:

- Presencia de los agentes sociales: se trataría de establecer diferentes grados de presencia de las organizaciones (sindicales, empresariales...) en mayor o menor proporción con respecto a la presencia de la Generalitat en las tres organizaciones comentadas. En este sentido, no se discute la existencia o no de esta representatividad, sino su mayor o menor peso en la composición de los órganos y/o en el ejercicio de funciones de los organismos

- Integración estructural: partiendo de un escenario con tres estructuras organizativas de diferentes naturalezas, se plantea la posibilidad de establecer diferentes niveles de integración estructural, concentrando las funciones ejercidas en un única institución o, por el contrario, mantener o ampliar el número de estructuras/instituciones establecidas.

Hay que advertir, sin embargo, que el hecho que estemos analizando tres instituciones diferentes (en lugar de una, cómo sería con el modelo teórico de los MOEs) hace incrementar notoriamente el número de posibles escenarios diferentes y, por lo tanto, hay que entender a los cuatro modelos que se presentarán acto seguido como los escenarios extremos en la aplicación de las dos variables (mayor/menor presencia de los agentes sociales y mayor/menor integración estructural), siendo conscientes por lo tanto que entre éstos existirían una pluralidad de modelos híbridos que también podrían ser objeto de estudio.

16 Tal y como se recoge en el preámbulo de la Ley 3/1997, de 16 de mayo, de creación del Consejo de Trabajo, Económico y Social de Catalunya. Cabe indicar que, pese a que esta ley se encuentra derogada por la Ley $7 / 2005$, de 8 de junio, es en aquella donde por primera vez tienen entrada dichas organizaciones.

17 Se constituye como tal en 1997 (http://web.pimec.org/es/pimec/qui-som).

18 Sería el órgano constituido mediante acuerdos interprofesionales que prevee el artículo 63 de la Ley 36/2011, de 10 de octubre, reguladora de la jurisdicción social. 
Así pues, los cuatro modelos que se proponen serían los siguientes:

\section{TABLA 1. MODELOS INSTITUCIONALES PROPUESTO}

\begin{tabular}{|c|c|c|c|}
\hline \multicolumn{4}{|c|}{ Mayor presencia agentes sociales } \\
\hline $\begin{array}{c}\text { Menor integración } \\
\text { estructural }\end{array}$ & $\begin{array}{l}\text { Modelo 3: } \\
\text { Tres organizaciones con personalidad } \\
\text { jurídica propia y menor presencia de los } \\
\text { agentes sociales en los órganos internos }\end{array}$ & $\begin{array}{l}\text { Modelo 4: } \\
\text { Una organización con personalidad } \\
\text { jurídica propia y menor presencia de los } \\
\text { agentes sociales en los órganos internos }\end{array}$ & $\begin{array}{l}\text { Mayor integración } \\
\text { estructural }\end{array}$ \\
\hline
\end{tabular}

Fuente: Elaboración propia

\subsection{MODELO 1: Mayor autonomía y preponderancia de los agentes sociales}

En este modelo se propone que el CTESC quede inalterable, tanto en términos de estructura, como de funciones y composición.

Por otra parte, habría que modificar la ley que regula el CRL para que se convierta en un organismo autónomo con personalidad jurídica propia y mejorar, así, su autonomía e independencia. En este sentido, en su Pleno sólo habría representación de las organizaciones sindicales y patronales más representativas, siguiendo al modelo del CTESC o de otros organismos autonómicos de participación institucional ${ }^{19}$ y, por lo tanto, desaparecería la representación de la Generalitat.

Por último y en relación con el TLC, se podría clarificar su naturaleza privada, derivada de la constitución del Tribunal como un acuerdo entre las organizaciones empresariales y sindicales más representativas y retirar, por lo tanto, la representación que actualmente tiene a la Generalitat de Catalunya en el patronato de la fundación.

\subsection{MODELO 2: Integración estructural y mayor preponderancia de los agentes sociales}

Este modelo supondría la integración en una única estructura organizativa de las tres organizaciones y con una preponderante presencia de las organizaciones empresariales y sindicales más representativas, de acuerdo con el punto anterior.

En este sentido y dado que el CTESC es la única institución configurada como órgano estatutario, se propondría ésta como organización acogedora de las otras dos y evitar, así, la necesidad de modificar el actual Estatuto de Autonomía de Catalunya. No obstante, sí que habría que modificar su ley y el resto de normas que se deriven de la misma.

Por otra parte, en el caso del CRL, se modificaría/derogaría su ley con el fin de integrar sus funciones de participación institucional en el CTESC (como de hecho, ya las tenía asignadas con la Ley 3/1997, de 16 de mayo), incorporando sus órganos de representación (Pleno) y de trabajo (comisiones y grupos de trabajo), así como el órgano ejecutivo. Las funciones de presidencia y vicepresidencia se agruparían en la de presidente y, eventualmente, de vicepresidente del CTESC.

En el caso del TLC y dado que su integración en el CTESC ya le conferiría, indirectamente, personalidad jurídica, quizás no sería necesaria la continuidad de la fundación privada que, tal y se comentó en el apartado 2.2.1, fue constituida de forma instrumental y con el objetivo de facilitar la actividad del Tribunal. Asimismo, se podría simplificar su estructura y reordenar sus funciones junto con las del CRL ${ }^{20}$. Ello no supondría la disolución del Tribunal

19 Ver el Consejo de Relaciones Laborales de Euskadi, http://web.crl-lhk.org.

20 Como por ejemplo la que confiere el artículo 82.3 del Estatuto de los Trabajadores, en relación con la inaplicación de convenios colectivos, como ya se verá más adelante. Hay que destacar que este precepto, junto a otros modificados por la Ley $3 / 2012$, de 6 de julio, de medidas urgentes para la reforma del mercado laboral, fue recurrido ante el Tribunal Constitucional por vulnerar el reconocimiento constitucional de la fuerza vinculante de los convenios colectivos (art. 37.1 CE), el ejercicio de la actividad sindical garantizado a través del reconocimiento constitucional de la libertad sindical (art. 28.1 CE), así como el derecho a la tutela judicial efectiva (art. 24 CE). Una vez admitido a trámite y a la espera de la oportuna sentencia, algunos organismos, como el Consejo de Garantías Estatutarias (Dictamen 5/2012) o autores como Eduardo Rojo Torrecillas (post en su blog “Mis dudas sobre la Ley 3/2012, los Dictámenes del Consejo de Garantías Estatutarias de la Generalitat, el recurso de inconstitucionalidad del PSOE e Izquierda Plural, y en general, nuevamente, sobre la reforma laboral (III)" apuntan a la inconstitucionalidad del artículo en cuestión. 
como tal, sino que se convertiría en un órgano del nuevo CTESC, habida cuenta de su singularidad relacionada con su constitución (derivada de acuerdo interprofesional) y composición (sin representación de la Administración en sus órganos internos). De hecho, se convertiría en un modelo muy similar de relación CRL-TLC como en el caso de Andalucía con el CARL-SERCLA ${ }^{21}$.

\subsection{MODELO 3: Mayor autonomía de las organizaciones y menor preponderancia de los agentes sociales}

Este modelo tomaría como estructura organizativa la descrita en el modelo 1, pero modificando la presencia de la Generalitat en los órganos internos de cada una de ellas.

En el caso del CTESC, la modificación vendría en la composición del Pleno que incluiría, de nuevo, el Grupo Cuarto, de representantes de la Generalitat, tal como se establecía en la Ley 3/1997, de 16 de mayo. Eso, por lo tanto, también afectaría a la composición de las diferentes comisiones y comportaría la modificación de la normativa existente al respecto.

Con respecto al CRL, la aplicación de este modelo significaría, básicamente, modificar su normativa reguladora con el fin de convertirlo en un organismo autónomo, dado que la representación actual de la Generalitat ya es un hecho y respeta la paridad entre las organizaciones sindicales y empresariales más representativas.

Por último y en relación con el TLC, supondría incrementar la presencia de la Generalitat en el patronato de la fundación en un miembro, con el fin de establecer una paridad entre los representantes de las diferentes organizaciones representadas, pero dejando inalterados la composición del resto de órganos para garantizar la autonomía colectiva

\subsection{MODELO 4: Integración estructural y menor preponderancia de los agentes sociales}

El último modelo que se propone se convierte en la confluencia entre las características estructurales del modelo 2 (un único organismo con personalidad jurídica propia) y los aspectos de representación del modelo 3 (mayor presencia de la Generalitat en los órganos de este único organismo).

En este sentido, el CTESC se convertiría en el único organismo con personalidad jurídica propia e incorporaría en su pleno y en el resto de órganos colegiados representantes de la Generalitat.

Con respecto al CRL, se integrarían sus funciones y órganos en la estructura actual del CTESC, que quedaría configurado como antes de la reforma del 2005.

Por último y en relación con el TLC, no haría falta la figura de fundación privada para desarrollar sus actividades ni, por lo tanto, alguno de los órganos actuales, básicamente aquellos vinculados directamente a la fundación (patronato y presidente). Si que se mantendría el Tribunal como órgano adscrito al CTESC, tal y como se apunta en el último párrafo del apartado 4.2.

\section{PROPUESTA DE MODELO INSTITUCIONAL}

De acuerdo con los objetivos recogidos en el apartado 1, el nuevo modelo institucional debería de dotarse de una estructura más racional y eficiente que se adapte mejor a la sociedad actual.

En este sentido, un modelo que apueste por una integración estructural de las actuales organizaciones supondría consolidar este modelo, pues se crearía un ente muy potente, con respecto a las funciones que asumiría y, por otra parte, posibilitaria la clara y única identificación institucional del espacio catalán de relaciones laborales.

Asimismo y en relación con el actual contexto económico, los poderes públicos tienen una mayor exigencia y responsabilidad ante la aplicación de criterios de eficacia y eficiencia en sus políticas, así como una mayor simplificación administrativa y de estructuras. Por lo tanto, parece ineludible llevar a cabo un análisis y valoración de las estructuras actuales y apostar, así, por una estructura organizativa que pueda ejercer plenamente sus competencias de una forma más ágil y con menos gasto económico. En esta línea, la reformulación del modelo estructural actual y la constitución de un único ente puede cumplir con estos objetivos.

Por último, hay que recordar que este modelo pivota necesariamente en la implicación de la Generalitat de Catalunya, dado que el Estatuto de Autonomía encarga su promoción, tal como hemos apuntado en el apartado

21 El Consejo Andaluz de Relaciones Laborales (CARL) se crea por la Ley 4/1983 de 27 de Junio, como órgano colegiado y tripartito, con representación de la administración andaluza y las organizaciones empresarials y sindicales más representativas y en él se encuentra adscrito el Servicio Extrajudicial de Resolución de Conflictos Laborales (SERCLA), de acuerdo con al disposición adicional tercera del Acuerdo Interprofesional para la Constitución del Sistema de Resolución Extrajudicial de Conflictos Colectivos Laborales de Andalucía. 
1. Por lo tanto, se podría pensar que su participación en el futuro modelo debería ser relevante y directa y con un claro rol de dinamizador de las relaciones laborales en Catalunya.

Por todo ello, se podria plantear la reformulación del espacio catalán de relaciones laborales mediante la creación de único organismo con presencia de las organizaciones empresariales y sindicales más representativas y de la Generalitat de Catalunya (modelo 4).

En este sentido y tal como se ha indicado en el punto 4.4, el CTESC ${ }^{22}$ posibilitaría la visualización institucional de este espacio, convirtiéndose en un organismo autónomo ${ }^{23}$, con personalidad jurídica propia, plena capacidad y autonomía orgánica y funcional, con adscripción en el Departamento de Empresa y Empleo y presupuesto propio dentro de los presupuestos de la Generalitat de Catalunya.

Este nuevo organismo supondría una racionalización de las estructuras administrativas actuales, pues se suprimirían diversos órganos, siendo un organismo más dinámico y próximo a la sociedad catalana. Asimismo, esta nueva estructura sería más eficiente y económica, dado que se crearían economías de escala ${ }^{24}$, producto de la integración de los servicios de soporte de los diferentes organismos integrados.

En relación con la composición del nuevo Consejo, se incluirían representantes de la Administración, de las organizaciones sindicales y empresariales más representativas, del sector agrario, marítimo pesquero y de la economía social, hecho que supondría reforzar la participación institucional del conjunto de las organizaciones más representativas y, sobre todo, de aquellas que tenían un papel más limitado (las organizaciones sectoriales del $\left(\operatorname{TESC}^{25}\right)$.

Con respecto a sus funciones, se añadiría a las que tiene actualmente asignadas el Consejo, las propias del TLC y del CRL, lo que supondría que el nuevo Consejo tuviera básicamente las siguientes:

- Hacer efectiva la participación institucional de las organizaciones sindicales y empresariales más representativas de Catalunya en la concertación social de las políticas laborales, junto con la Generalitat de Catalunya.

- Impulsar el diálogo social y asistir en la articulación de un marco de negociación colectiva catalán.

- Fomentar la resolución extrajudicial de los conflictos laborales, mediante la mediación y el arbitraje, mediante el TLC.

- Dictaminar sobre las normas jurídicas que regulen materias socioeconómicas, laborales y de empleo competencia de la Generalitat.

- Asesorar al Gobierno de la Generalitat, mediante estudios, informes o propuestas, sobre materias socioeconómicas, laborales y de empleo, así como informar sobre la situación socioeconómica y laboral de Catalunya.

\section{ESTRATEGIA DE IMPLEMENTACIÓN}

Una vez descrito el modelo institucional propuesto para el espacio catalán de relaciones laborales, pasaríamos a describir lo que sería la hoja de ruta para llevarlo a la práctica. Este plan se estructuraría en tres dimensiones: la creación del nuevo ente y su estructuración, la redefinición de sus procesos de trabajo y la concreción de los recursos económicos y humanos que dispondria.

No obstante y de forma previa a avanzar en cómo implementar esta propuesta, hay que comentar brevemente la situación actual hacia la acreditación de la representatividad de las organizaciones empresariales, dado que su resolución puede comprometer la aplicación de este modelo.

A diferencia del que sucede en la determinación de las organizaciones sindicales más representativas, a efectos de su representación y participación institucional, la acreditación de la consideración de organización empre-

22 Tal y como se ha comentado anteriormente, se plantea escoger este organismo como ente único (en lugar de crear uno nuevo), con el fin de no modificar el actual Estatuto de Autonomía. Eso comporta, lógicamente, no alterar su denominación, aunque tuviera unas funciones más amplías a las actuales.

23 Con este cambio de naturaleza jurídica, el Consejo posibilita la adscripción de personal funcionario en su plantilla.

24 La integración de unidades u órganos como las indicadas, posibilitaría la aplicación del criterio de interdependencias de escala enunciado por Mintzberg (Mintzberg, 1998: 155-156), dado que se podría disponer de un único responsable en materia de recursos humanos (en vez de uno en cada organismo), en gestión económica,...ello generaría un ahorro económico (input), manteniendo e, incluso, mejorando, el servicio ofrecido (output). Este criterio también se aplicaría a los cargos representativos/ejecutivos (Presidencia, Secretaria Ejecutiva).

25 Organizaciones representativas del sector agrario, marítimo pesquero y de la economía social. 
sarial más representativa se encuentra pendiente de desarrollo normativo, pues sólo existe como regulación la disposición adicional $6^{\mathrm{a}}$ del Estatuto de los Trabajadores, que establece que se entiende que disfrutan de esta capacidad representativa las asociaciones empresariales que tienen el 15\% o más de las empresas y trabajadores de la comunidad autónoma. Así, nos encontramos con que actualmente no hay ningún procedimiento con el fin de acreditar que una organización empresarial dispone de este porcentaje de empresas y trabajadores (hay que indicar que el desarrollo legal o reglamentario de esta disposición lo tendría que llevar a cabo el Estado, que es quien tiene la competencia exclusiva en legislación laboral). En Catalunya, esta representatividad se ha concretado en la mayor parte de los órganos de representación empresarial con un cierto consenso entre la patronal Fomento y PIMEC, las cuales ostentan actualmente la condición de organizaciones empresariales más representativas. No obstante, una sentencia del Tribunal Supremo ${ }^{26}$ ha puesto fin a un largo procedimiento judicial y ha confirmado una sentencia anterior del Tribunal Superior de Justicia de Catalunya, por la cual otorgaba a la patronal FEPIME la consideración de organización empresarial más representativa en Catalunya.

Por lo tanto, antes de entrar en la reformulación del modelo institucional del espacio catalán de relaciones laborales, sería interesante poder aclarar como determinar las organizaciones empresariales más representativas que tienen que formar parte de este modelo. Se podrían proponer tres posibles acciones a realizar por parte de la Generalitat en este sentido:

1. Instar en el Estado a que regule un procedimiento que concrete como se acredita esta representatividad.

2. Favorecer un acuerdo entre las organizaciones empresariales que posibilite una interlocución y participación institucional unitaria con la Generalitat (es decir, como si efectivamente sólo hubiera una única organización empresarial).

3. Impulsar la aprobación de una ley de participación institucional (como ya tienen en Galicia, Cantabria o las Islas Baleares) que, aparte de regular el marco de la participación institucional de las organizaciones sindicales y empresariales más representativas de Catalunya (ejercicio de la participación, ámbito subjetivo, compensaciones económicas,...), establezca, dentro de las competencias de organización de las instituciones de autogobierno que tiene la Generalitat, el procedimiento para acreditar la representatividad de las organizaciones empresariales, de acuerdo con los criterios de la disposición adicional $6^{\text {a }}$ del Estatuto de los Trabajadores, a los efectos de su participación institucional.

\subsection{Creación y estructuración del nuevo ente}

Una vez realizada esta observación, hay que indicar que la aplicación de este modelo requiere, sin duda, de un gran pacto político entre las diferentes formaciones políticas a fin de que el nuevo CTESC se convierta en una institución sólida y legitimada. También se necesitará un gran trabajo de consenso con las organizaciones empresariales y sindicales más representativas (de aquí la importancia de poder acreditar claramente la representatividad de éstas), así como de las otras organizaciones que integrarían el Consejo. Así, se podría promover la firma de un documento de base, como un "Acuerdo por el impulso de espacio catalán de relaciones laborales", que recogiera el compromiso de las partes en promover este espacio y redefinir su modelo institucional.

Por lo tanto, habría que llevar a cabo las siguientes acciones²7:

- Firma del Acuerdo por el impulso de espacio catalán de relaciones laborales.

- Aprobación de la Ley de Participación Institucional.

- Denuncia del convenio de colaboración entre el Departamento de Empresa y Empleo y las organizaciones Fomento, CCOO y UGT, por el cual se establece la financiación de la TLC.

- Disolución de la fundación privada Tribunal Laboral de Catalunya.

- Aprobación de la nueva ley del Consejo de Trabajo, Económico y Social de Catalunya, que comportaría la derogación de la Ley 1/2007, de 5 de junio, del Consejo de Relaciones Laborales y la Ley 7/2005 de 8 de junio, del Consejo de Trabajo, Económico y Social de Catalunya.

26 Sentencia del Tribunal Supremo 6179/2012, Sala del Contencioso, Sección 4, sobre recurso de casación $4332 / 2011$.

27 Aceptando la dificultad en realizar estas acciones, cabe indicar que, en el caso de la disolución de la fundación privada (que no del Tribunal Laboral de Catalunya), sería una medida recogida en el Acuerdo por el impulso de espacio catalán de relaciones laborales, en tanto en cuanto debería efectuarse por las mismas organizaciones que la crearon. 
- Aprobación de un Decreto de despliegue de la nueva Ley del Consejo, que al mismo tiempo regule el funcionamiento interno de lo mismo.

En relación con la estructura organizativa del nuevo Consejo, se propone tres órganos unipersonales de tipo representativo (Presidencia), directivo (Vicepresidencia) y ejecutivo (Secretaría General), un órgano ejecutivo colegiado (Comisión Ejecutiva), un órgano de representación (Pleno) y los órganos de trabajo (comisiones y grupos de trabajo). Asimismo, también dispondría de un cuerpo de mediadores y un cuerpo de árbitros.

- Presidencia: Nombramiento y separación por parte del Gobierno, a propuesta conjunta del consejero del Departamento de Empresa y Empleo y de Economía y Conocimiento y con el apoyo de dos tercios de los miembros del Pleno. Sus funciones serían esencialmente de representación institucional.

- Vicepresidencia: su nombramiento lo acuerda el Gobierno a propuesta del Presidente del Consejo. Sus funciones serían de dirección de las actuaciones del Consejo, así como de representación legal del mismo.

- Secretaría General: sería el órgano que gestiona y garantiza el funcionamiento ordinario del Consejo. Este puesto, de carácter técnico, sería funcionarial, con provisión mediante el sistema de libre designación, siendo nombrado y separado por el consejero de Empresa y Empleo.

- Pleno: Bajo la dirección del presidente, integra los treinta y seis miembros del Consejo: diez miembros en representación de la Generalitat (incluye el vicepresidente, el secretario general y ocho expertos en materias del Consejo), diez miembros en representación de las organizaciones sindicales, diez miembros en representación de las organizaciones empresariales, seis miembros en representación del sector agrario, pesquero marítimo y de la economía social. De sus funciones destacaríamos la aprobación del presupuesto anual, del plan anual de trabajo, de los informes, estudios, dictámenes y propuestas, así como conocer las líneas estratégicas de la Generalitat en materia laboral y proponer al presidente del Consejo.

- Comisión Ejecutiva: Estaría formada por ocho miembros del Consejo: dos miembros en representación de la Generalitat (vicepresidente y secretario general), dos miembros en representación de las organizaciones sindicales, dos miembros en representación de las organizaciones empresariales y dos miembros en representación del sector agrario, pesquero marítimo y de la economía social. Como funciones, tendríamos la aprobación del anteproyecto de presupuesto, del proyecto de memoria anual o la asignación y coordinación de los trabajos las comisiones y los grupos de trabajo.

- Comisiones y grupos de trabajo: las comisiones son los órganos encargados de la elaboración de las propuestas de dictámenes, estudios e informes, así como de impulsar la participación institucional de las organizaciones más representativas. Se encuentran compuestas por doce miembros, de forma proporcional en los diferentes grupos y están dirigidas por el vicepresidente y asistidas por el titular de la secretaría general. Los grupos de trabajo, a diferencia de las comisiones, tendrían un carácter más técnico y temporal y, por lo tanto, se crearían y se suprimían por acuerdo del Pleno.

- Cuerpo de mediadores: está constituido por las personas designadas por las organizaciones empresariales y sindicales más representativas, así como por la Generalitat, con el fin de formar parte en las comisiones de mediaciones.

- Comisiones de mediaciones: son los órganos competentes en el intento de resolución de un conflicto laboral. Están compuestas por cinco miembros: dos en representación de las organizaciones empresariales más representativas, dos en representación de las organizaciones sindicales más representativas y uno en representación de la Generalitat, que ejercerá de secretario de la comisión y validará, si procede, los acuerdos alcanzados.

- Cuerpo de árbitros: está constituido por los árbitros designados por las organizaciones empresariales y sindicales más representativas, así como los árbitros designados por la Generalitat. Son asignados a propuesta conjunta de las partes en conflicto, las cuales se someten a su decisión o laudo, siendo, por lo tanto, vinculante para las mismas.

Esquema organizativo

- Presidencia

- Vicepresidencia

- Secretaría General

- Pleno

- Diez miembros en representación de la Generalitat

GESTIÓN Y ANÁLISIS DE POLÍTICAS PÚBLICAS, Nueva Época, nº 11 enero-junio 2014 ISSN: 1989-8991 - DOI: 10.24965/gapp.v0i11.10175 
- Diez miembros en representación de las organizaciones empresariales más representativas.

- Diez miembros en representación de las organizaciones sindicales más representativas.

- Seis miembros en representación del sector agrario, pesquero marítimo y de la economía social

- Comisión Ejecutiva

- Comisiones

- Grupos de trabajo

- Cuerpo de mediadores

- Comisiones de mediaciones

- Cuerpo de árbitros

\subsection{Procesos de trabajo}

El encaje de las funciones del anterior CTESC en la estructura del nuevo no nos supondría una gran dificultad, pues ya tenían una gran similitud tanto en composición, como en la organización de los respectivos órganos (pleno, comisiones y grupos de trabajo). Así pues, sólo haría falta simplificar y redefinir, en todo cas, el número y el ámbito de actuación de los diferentes órganos de trabajo (en este sentido, sería volver a la composición y funciones del CTESC anterior a la reforma del 2005).

No obstante, en el caso del TLC haría falta una reflexión adicional, pues aparte que actualmente no tiene representación a la Generalitat en sus órganos (a excepción del Patronato de la Fundación), el actual marco normativo ha atribuido al CRL funciones en materia de arbitraje que, hasta la fecha, ejercía de forma casi exclusiva el TLC.

Por otro lado, la reciente modificación del Estatuto de los Trabajadores establece, en el artículo 82.3, que ante la falta de acuerdo entre empresario y trabajador en la modificación de determinadas condiciones de trabajo establecidas en el convenio colectivo aplicable, y una vez agotadas las instancias previas, cualquiera de las partes puede someter la resolución de esta discrepancia a la Comisión Consultiva Nacional de Convenios Colectivos o su análogo a nivel de comunidad autónoma (en el caso de Catalunya, el CRL). Por lo tanto, en el decreto de desarrollo y organización del nuevo CTESC habrá que regular el procedimiento a seguir ante estas peticiones.

Asimismo y con respecto a la gestión de las mediaciones y arbitrajes que realizaba el TLC, serían siendo una tarea que seguirían realizando las personas nombradas por las organizaciones empresariales y sindicales más representativas, con la inclusión en dichas comisiones de un representante de la Generalitat que, aparte de colaborar en la mediación propiamente dicha, podrá ejercer el control de legalidad sobre los acuerdos. Por otra parte y con respecto a la necesidad de asistencia ante un aspecto técnico que haya surgido en las mediaciones, las comisiones de mediaciones lo podrán trasladar a la respectiva comisión del CTESC para su estudio e informe.

\subsection{Recursos humanos y económicos}

Una vez creado legalmente y establecida su estructura básica, hay que dotar al nuevo ente de los medios humanos y económicos necesarios con el fin de poder hacer efectivas sus funciones.

En relación con el presupuesto, habrá que analizar las necesidades del nuevo CTESC. Es de prever que la nueva estructura sea más eficiente $y$, por lo tanto, que el presupuesto del ente se pueda situar por debajo de la suma de los presupuestos de las tres organizaciones actuales, que en el 2011 se situaría en un mínimo de 3.887.394,20 $€^{28}$. En este sentido, el aplanamiento de estructura y la reducción de cargos derivados de la integración de las tres organizaciones, añadido a la reorganización interna de las unidades de soporte al nuevo Consejo, adscritas a la Secretaría General, como recursos humanos o gestión económica, tiene que posibilitar este ahorro económico ${ }^{29}$. Tal como se ha indicado anteriormente, este organismo autónomo dispondría de personalidad jurídica propia y presupuesto propio dentro de los presupuestos de la Generalitat de Catalunya.

28 El presupuesto del CRL se encuentra agregado dentro de la Secretaría de Empleo y Relaciones Laborales y, por lo tanto, no se puede concretar. No obstante, el presupuesto del TLC fue de 946.336,90€ (Resolución EMO/1705/2012, de 11 de julio), y el del CTESC fue de 2.941.057,30€ (Ley 6/2011, del 26 de julio, de presupuestos de la Generalitat de Catalunya para el 2011).

29 Este ahorro económico vendría derivada, fundamentalmente, por las economías de escala, tal y como se indica en la nota 25. 
Con respecto a los recursos humanos, habrá que redefinir la relación de puestos de trabajo (RLT), partiendo de las plantillas de las instituciones que se han integrado en el Consejo. Esta RLT estaría, pues, inicialmente compuesta por personal funcionario (proveniente del Consejo de Relaciones Laborales) y laboral (proveniente del Tribunal Laboral de Catalunya y del Consejo de Trabajo, Económico y Social).

\section{REFERENCIA BIBLIOGRÁFICAS Y DOCUMENTALES}

- Acuerdo Interprofesional de Catalunya. http://portaldogc.gencat.cat/utilsEADOP/AppJava/PdfProviderS ervlet?documentld $=66139 \&$ type $=01 \&$ language $=$ es_ES

- Acuerdo Interprofesional para la Constitución del Sistema de Resolución Extrajudicial de Conflictos Colectivos Laborales de Andalucía. http://www.juntadeandalucia.es/empleo/carl/portal/c/document_library/ get_file?uuid=599acf72-bbcf-444d-beoc-d8ca2cd924a3\&groupld=10128

- Blog de Eduardo Rojo Torrecillas. http://www.eduardorojotorrecilla.es/2012/10/mis-dudas-sobre-la-ley32012-los_2539.html

- Convenio de col·laboración entre el Departamento de Empresa y Empleo y la Fundación Privada Tribunal Laboral de Catalunya, de 12 de mayo de 2012 http://www20.gencat.cat/docs/empresaiocupacio/01\%20-\%20 Informacio\%20Departamental/Documents/04\%20-\%20Tauler\%20informacio\%20publica/Convenis\%20de\%20 collaboracio/Arxius/Conveni_Tribunal_Laboral_Catalunya.pdf

- Comisión para la Reforma de las Administraciones Pública (CORA). http://www.lamoncloa.gob.es/NR/ rdonlyres/BDCE2E62-85EE-4D59-B72B-79888906B581/0/InformeCORAPDF.PDF

- Decreto 352/2011, de 7 de junio, de reestructuración del Departamento de Empresa y Empleo, https:/l www.gencat.cat/eadop/imagenes/5897/11153112.pdf

- Dictamen 5/2012, de 3 de abril, del Consejo de Garantías Estatutarias, con respecto al Real decreto-ley 3/2012, de 10 de febrero, de medidas urgentes para la reforma del mercado laboral. http://www.cge.cat/ admin/uploads/docs/20130325194909-2.pdf

- Estatuto de Autonomía de Catalunya, http://www.gencat.cat/generalitat/cas/estatut

- Estatuto de los Trabajadores, http://www.empleo.gob.es/es/sec_leyes/trabajo/estatutoo6/

- Guía rápida del Tribunal Laboral de Catalunya, http://www.tribulab.cat/wp-content/uploads/2014/02/TLC2013CATALAx.pdf

- Ley 4/1983 de 27 de Junio, del Consejo Andaluz de Relaciones Laborales. http://www.juntadeandalucia.es/empleo/carl/portal/c/document_library/get_file?uuid=860380 cb-9b93-45e7-bag96864 fc0 400 f 3 \&groupld $=10128$

- Ley 3/1997, de 16 de mayo, de creación del Consejo de Trabajo, Económico y Social de Catalunya. http:/l portaldogc.gencat.cat/utilsEADOP/AppJava/PdfProviderServlet?documentld=144911\&type=01\&languag e=es_ES

- Ley 7/2005, de 8 de junio, del Consejo de Trabajo, Económico y Social de Catalunya. http://portaldogc. gencat.cat/utilsEADOP/AppJava/PdfProviderServlet?documentld=386810\&type=01\&language $=e s \_E S$

- Ley $1 / 2007$, del 5 de junio, del Consejo de Relaciones Laborales. http://www20.gencat.cat/portal/site/ portaldogc/menuitem.c973d2fc58aa0083e4492d92bocoe1ao/?vgnextoid=485946a6esdfe210VgnVCM10 0000oboc1eoaRCRD\&applnstanceName=default\&action=fitxa\&documentld=444917

- Ley $17 / 2008$, de 29 de diciembre, de participación institucional de las organizaciones sindicales y empresariales más representativas de Galicia. http://www.boe.es/boe/dias/2009/03/16/pdfs/BOE-A-2009-4370.pdf

- Ley de Cantabria 4/2009, de 1 de diciembre, de Participación Institucional de los Agentes Sociales en el Ámbito de la Comunidad Autónoma. http://www.boe.es/boe/dias/2010/01/21/pdfs/BOE-A-2010-929.pdf

- Ley 6/2011, del 26 de julio, de presupuestos de la Generalitat de Catalunya para el 2011. http://www15. gencat.cat/ecofin_wpres11/pdf/ART_CAS.pdf

- Ley $2 / 2011$, de 22 de marzo per la que se regula la participación institucional de las organizaciones empresariales y sindicales más representativas de la comunidad autónoma de las Illes Balears. http://www.boe. es/boe/dias/2011/04/25/pdfs/BOE-A-2011-7338.pdf 
- Ley 36/2011, de 10 de octubre, reguladora de la jurisdicción social. http://www.boe.es/buscar/act. php?id=BOE-A-2011-15936

- Mintzberg, H. (1988), La Estructuración de las organizaciones. Barcelona, editorial Ariel.

- Ramió, C.; Salvador, M. "Los modelos de orientación estratégica (MOEs): una adaptación del enfoque estratégico para el diseño organizativo en las Administraciones públicas". GAPP, n. 16, diciembre 1999 (89-105) http://revistasonline.inap.es/index.php?journal=GAPP\&page=article\&op=viewFile\&path\%5B\%5D $=199 \&$ path\% 5 B\% $5 \mathrm{D}=199$

- Resolución EMO/1705/2012, de 11 de julio, por la que se da publicidad a las subvenciones concedidas por el Departamento de Empresa y Empleo durante el año 2011. http://www20.gencat.cat/portal/site/portaldogc/menuitem.c973d2fc58aa0083e4492d92bocoe1ao/?vgnextoid=485946a6esdfe210VgnVCM1000000 boc1eoaRCRD\&applnstanceName $=$ default\&action $=$ fitxa\&documentld $=615880$ \&language $=e$ _ES

- Sentencia del Tribunal Supremo 6179/2012, Sala del Contencioso, Sección 4, sobre recurso de casación 4332/2011. http://www.poderjudicial.es/search/doAction?action=contentpdf\&databasematch=TS\&refere nce $=6515047 \&$ links $=\% 224332 / 2011 \% 22 \&$ optimize $=20121016 \&$ publicinterface $=$ true

- Web del Consejo Andaluz de Relaciones Laborales. http://www.juntadeandalucia.es/empleo/carl/

- Web del Consejo de Relaciones Laborales (CRL). http://crl.gencat.cat

- Web del Consejo de Relaciones Laborales de Euskadi. http://web.crl-lhk.org

- Web del Consejo de Trabajo, Económica y Social de Catalunya (CTESC). http://www.ctesc.cat

- Web de la Pequeña y la Mediana Empresa de Catalunya (PIMEC). http://web.pimec.org

- Web del Tribunal Laboral de Catalunya (TLC). http://www.tribulab.cat 